\title{
Capacity and Error Rate Analysis of SIMO Satellite Systems over Ka Band at Venezuelan Amazonian
}

\author{
Gustavo Guedez ${ }^{1,}$, Qiang Gao ${ }^{1, b}$ and María Guarirapa ${ }^{1, c}$ \\ ${ }^{1}$ School of Electronic and Information Systems, Beihang University, Beijing,China \\ agguedezcarias@hotmail.com, bgaoqiang@buaa.edu.cn, catenea121@hotmail.com
}

Keywords:SIMO, Capacity, $\mathrm{E}_{\mathrm{b}} / \mathrm{N}_{0}, \mathrm{BER}, \mathrm{Ka}$ Band.

\begin{abstract}
Satellite systems progressively operate in higher frequencies, such as the Ka bands. The signals in Ka band system are seriously affected by the environment constraint. A significant percentage of the VENESAT-1 (Venezuelan Satellite) footprint has the coverage service area over the Amazonian. Due to the large distances from the satellite and the ground station, the signals strength decrease exponentially. Thus, the link quality is degraded which leads to unacceptable error rate and lower system capacities. In order to overcome this problem, the diversity concept can be used. The diversity principle is based on the transmission of multiple copies of the same signal, which can be combined at the receiver or be selected the best copy of the signal. The research aim is to simulate the performance of the Venezuelan Satellite with several receive antennas similar to a SIMO system. The main purpose, is to analyze the system capacity and bit error rate of $\mathrm{BER}=10^{-6}$ with lower values of $\mathrm{E}_{\mathrm{b}} / \mathrm{N}_{0}$ and compare the results with SISO performance.
\end{abstract}

\section{Introduction}

The Amazonian has innumerable natural wonders and its rivers represent the biggest fresh water reserve of the world. The construction of wire guide communication system is not possible, because international environment regulation protect the Amazonian. The satellite is the best method to provide communications services at the Amazonian area. However, the Ka band can suffer significant degradation due to the environment. The signal strength on satellite system varies, because several environment conditions and in some cases the link is not able to deliver data successfully. This produce unacceptable radio link failure $[1,2]$. The diversity principle can be useful to combat the problem. Diversity relies on the transmission of multiple copies from the same signal. The receiver can combine the signals or select the best copy of them. Thus, even if one copy of the signal has not sufficient quality, the probability that all the copies has poor quality is very low [3]. According to the generation of the copies exist different kind of diversity. The signal copies can be generated transmitting the same signal multiple times (time diversity) or using different frequencies of the spectrum (frequency diversity). However, in the Venezuelan Satellite system can be useful to explore the communication link employing multiple antennas (space diversity). The Venezuelan Satellite in Ka band has a single antenna working in a single polarization. The Venezuelan Satellite system can be modeled as a SIMO system. The diversity provide link quality and stability which leads to improve the performance of the communication system [3]. To be effective the diversity must generate copies of the signal independent to each other, in order to minimize the probability that all the signals face simultaneously bad propagation conditions. The diversity gain have to be analyzed over the transmission channel. Normally for satellite communication, the channel can be modelled as the Rice distribution [3, 4]. Rice distribution consist of communication paths which as a strong line of sight (LOS) component combined with several weaker multipath components [4]. The multipath component can be modelled as a Rayleigh distribution [4]. 


\section{System Model}

\subsection{VENESAT-Multiple Receive Earth Station}

Consider the scheme shown in the Fig.1. The Venezuelan satellite transmit to several earth station. The earth station has fixed antennas and the satellite is only able to transmit in one polarization.

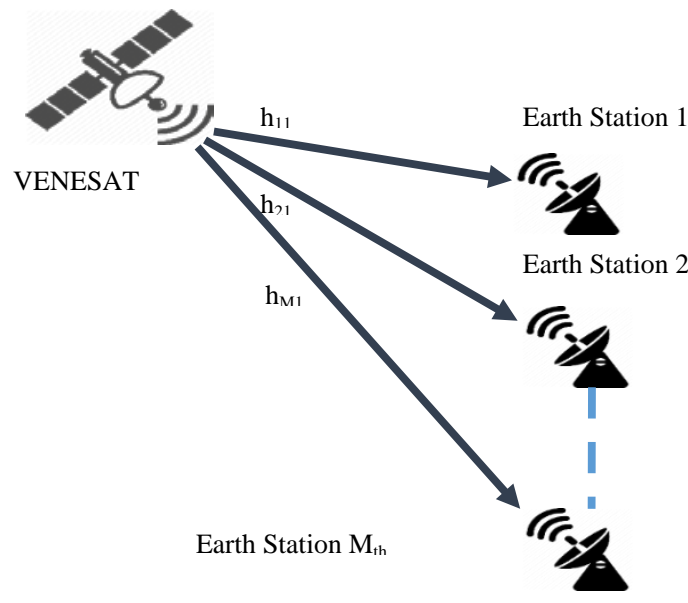

Fig.1 VENESAT transmission to Multiple Earth Stations

The channel between the Venezuelan satellite and the earth station can be modelled as Mx1 SIMO channel. Where $h_{M 1}$ is the channel between the satellite antenna and the $\mathrm{M}_{\text {th }}$ earth station. The received signal at the earth $[3,6]$

$$
\left(\begin{array}{c}
\mathrm{y}_{1} \\
\mathrm{y}_{2} \\
\vdots \\
\mathrm{y}_{\mathrm{M}}
\end{array}\right)=\left(\begin{array}{c}
\mathrm{h}_{11} \\
\mathrm{~h}_{21} \\
\vdots \\
\mathrm{h}_{\mathrm{M} 1}
\end{array}\right)(\mathrm{x})+\left(\begin{array}{c}
\mathrm{n}_{1} \\
\mathrm{n}_{2} \\
\vdots \\
\mathrm{n}_{\mathrm{M}}
\end{array}\right)
$$

$Y=H x+n$

Where $y=\left[\mathrm{y}_{1}, \mathrm{y}_{2}, . ., \mathrm{y}_{\mathrm{M}}\right]^{\mathrm{T}}$ is an $\mathrm{Mx} 1$ vector of the received signal, $\mathrm{x}$ is the signal transmitted at one polarization and $\mathrm{n}=\left[\mathrm{n}_{1}, \mathrm{n}_{2}, \ldots, \mathrm{n}_{\mathrm{M}}\right]^{\mathrm{T}}$ is an $\mathrm{Mx} 1$ vector of Gaussian noise with $\mu=0$ and variance $\sigma 2$.

\section{Channel Model}

\subsection{A. Scintillation and multipath effects}

The scintillations is defined by the magnitude of the refractive index in the propagation path, crossing the troposphere. The scintillations is related to the frequency and the path length. From Experimental data the monthly-averaged of the wet term (radio refractivity) "Nwet" can be obtained $[7,8,9,10]$. The main parameters to calculate the multipath are given by

$$
\text { oref }=3.6 * 10^{-3}+10^{-4} * N \operatorname{wet}(3)
$$


The effective path length with $\mathrm{hL}=1000 \mathrm{~m}$

$$
L=\frac{2 * h L}{\sqrt{\sin ^{2} \theta+2.35 * 10^{-4}+\sin \theta}}(4)
$$

The antenna effective diameter:

$$
D e f f=\sqrt{\eta} * D(5)
$$

The antenna averaging factor is given by:

$$
\begin{aligned}
& g(x)=\sqrt{\left(3.86\left(x^{2}+1\right)^{\frac{11}{12}} \sin \left[\frac{11}{6} \tan ^{-1}\left(\frac{1}{x}\right)\right]-7.08 x^{\frac{5}{6}}\right)}(6) \\
& x=1.22 \operatorname{Deff}^{2}\left(\frac{f}{L}\right)(7)
\end{aligned}
$$

The standard deviation is:

$$
\sigma=\sigma r e f f^{\frac{7}{12}}\left(\frac{g(x)}{\sin \theta^{1.2}}\right)(8)
$$

Calculating the attenuation, exceeded for $\mathrm{p} \%$. The parameter p between $0.01 \%$ and $50 \%$ $A(p)=a(p) * \sigma(9)$

$a(p)=-0.061[\log 10(p)]^{3}+0.072[\log 10(p)]^{2}-1.71[\log 10(p)]+3.0(10)$

\subsection{Free Space Loss Model}

The free space SIMO model is based on the line of sight (LOS) component of the fading channel. The channel model can be represented [6].

$$
\boldsymbol{H}_{i j}=\boldsymbol{\alpha}_{i j} * \boldsymbol{e}^{\left(-j k_{0} f_{c} r_{i}\right)}(11)
$$

The term $\mathrm{f}_{\mathrm{c}}$ is the carrier frequency, $\mathrm{r}_{\mathrm{i}}$ is the distance between the satellite and the $\mathrm{i}_{\text {th }}$ earth station receive antenna, $\mathrm{k}_{0}=\left(2 * \pi / \mathrm{c}_{0}\right)$ is the wave number and $\mathrm{c}_{\mathrm{o}}$ is the light speed.The phase $\varphi$ is assumed equal to zero and $\alpha$ (attenuation) is approximated to a constant [6].

$$
\alpha_{i j}=\frac{1}{2 k_{0} f_{c} r_{i}} * e^{(-j \varphi)}
$$

\subsection{Analytical SIMO Model}

The channel model is a combination of two parts: the LOS component and non LOS component (multipath component) [2, 5].For satellite communication with fixed antennas the multipath effect is minimum. For a narrow band system without Channel State Information at the Transmitter (CSIT) the capacity for SIMO can be calculated [3].

$$
C=\log _{2}(1+\rho *\|h\|)
$$


In satellite communication for BPSK and QPSK signal the maximum SNR is compute [11].

$S N R=\sqrt[2]{\frac{E_{b}}{N_{0}}}$

The probability of obtain an error due the noise [11]

$P_{e}=\frac{1}{2} \operatorname{erfc}\left(\sqrt{\frac{1}{2} \frac{E_{b}}{N_{0}}}\right)$

\section{Simulation Results}

\subsection{Simulation of Scintillation and multipath effects}

For systems with antennas at low elevation angles, multiple indirect paths are normally produced between the transmitter and receiver $[2,11]$. This is caused by the reflections of the signals with the surrounding objects. The signal reflection will cause an addition of signals (interference). The simulation result for Scintillation and Multipath Effects can be seen in Fig. 2.

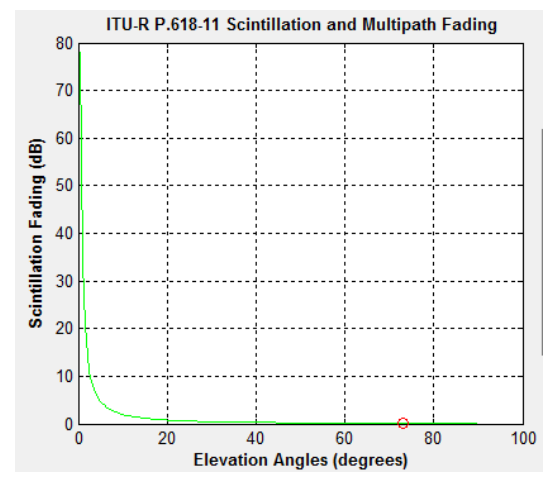

Fig. 2 Simulation of scintillation and multipath effects.

\subsection{Ergodic Capacity and Bit Error Rate}

The ergodic capacity and bit error rate (BER) assume signals transmitted over a Rice channel with BPSK modulation. The Rice channel is composed by two components the strong LOS component and the multipath weaker components. The simulation result are shown in Fig. 3.

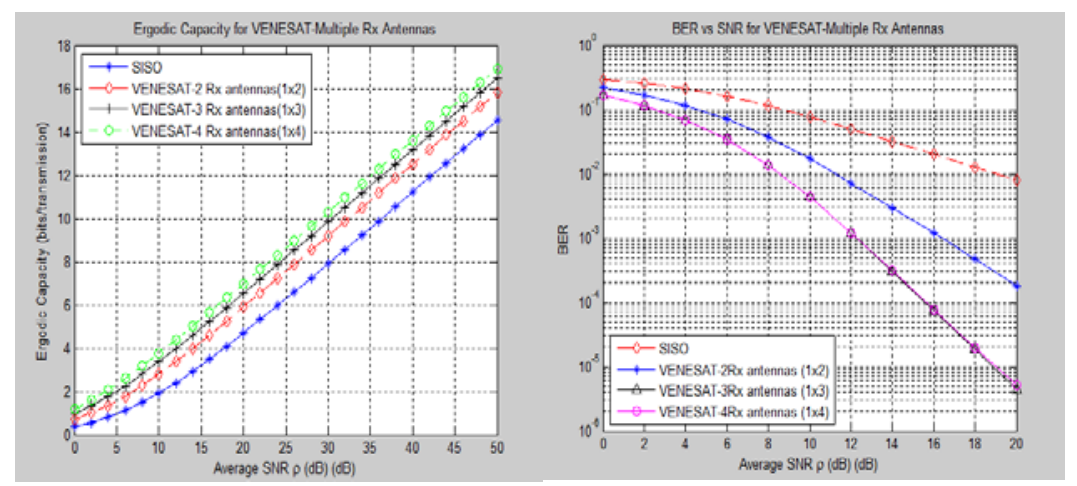

Fig. 3 Simulation of Ergodic Capacity and BER. 
The Fig. 3 shows the ergodic capacity increases according to the number of receive antennas. The BER has animportant improve when additional antennas are added in comparison with SISO system. Three receive antennas and four receive antennas has a similar performance.The diversity is a process of diminishing return, in others words, the benefit to add a fourth antenna to a three antenna system is smaller than add a second antenna to the SISO system [3].

\section{Conclusion}

Satellite communication systems operating at Ka band frequencies at the Amazon follow a Rice distribution. The scintillation and multipath effects can be neglected because the Venezuelan latitude is close to equator and for that reason the elevation angles are high enough to avoid obstacle between the satellite and the field of view of the receive antennas. The channel can be approximated to the free space model, because the multipath effects are not significant compare with the antenna main lobe receive signal. For the fixed antennas earth stations, the multipath effect is very low, especially in ground stations with big diameter antennas. Multiple receive antennas improve the ergodic capacity of the system compare with the SISO scenario. The improvement allow to the service providers increase the quality of the services in the remote areas located at the Venezuelan Amazonian.Increasing the number of receive antenna increase the system reliability due is decreased the probability of error. The SIMO model can be used as a mitigation technique, using the diversity gain to compensate some levels of rain attenuation.

\section{References}

[1] R. T. Schwarz, A. Knopp, D Ogerman, C.A. Hofmann and B. Lankl, “Optimun Capacity MIMO Satellite Link for Fixed and Mobile Services,” Proceedings IEEE Workshop on Smart Antennas, 2008, vol. 1 pp. 209-216.

[2] ITU R P.618-11, "Propagation data and prediction methods required for the design of Earth-space telecommunication systems” (2013).

[3] T.Brown, E. De Carvalho, P. Kyritsi “Practical Guide to the MIMO Radio Channel” New York: WILEY 2012.

[4] M.Simon, M.Alouini “Digital Communication over Fading Channels” New York: WILEY 2012.

[5] Loo, C. “A statistical Model for a Land Mobile Satellite Link," IEEE transaction of vehicular Technology, 1998 vol. 34 pp. 122-127.

[6] Ramoni Adeogun, "Capacity and Error Rate Analysis of MIMO Satellite Communication Systems in Fading Scenarios,” International Journal of Electrical and Computer Engineering (IJECE), August 2014, vol. 4 pp. 612-622.

[7] ITU-R P.676-10, “Attenuation by atmospheric gases” (2013).

[8] ITU-R P.835-5, “Reference standard atmospheres”(2012).

[9] ITU-R P.1510, “Annual Mean Surface Temperature” (2001).

[10] ITU-R P.453-8 “The radio refractive index: its formula and refractivity data” (2001).

[11] D. Rodis, “Satellite Communications,” New York: McGraw-Hill 2006. 